\title{
Oscillatory Propagation of a Rich Premixed Spray Flame
}

\author{
L.S. Kagan $^{1}$, J.B. Greenberg ${ }^{2}$ and G.I. Sivashinsky ${ }^{3}$ \\ ${ }^{1}$ Sackler Faculty of Exact Sciences, School of Mathematical Sciences Tel Aviv University, \\ Tel Aviv 69978, Israel \\ ${ }^{2}$ Faculty of Aerospace Engineering Technion - Israel Institute of Technology, Haifa 32000, Israel
}

\begin{abstract}
Experimental evidence points to a rich variety of physical scenarios that arise when a laminar flame propagates through a pre-mixture of evaporating liquid fuel and a gaseous oxidant. In this paper new results of time-dependent numerical simulations of rich off-stoichiometric spray flame propagation in a two-dimensional channel are presented. A constant density model is adopted, thereby eliminating the Darrieus-Landau instability. It is demonstrated that there exists a narrow band of vaporization Damkohler numbers (the ratio of a characteristic flow time to a characteristic evaporation time) for which the flame propagation is oscillatory. For values outside this range steady state propagation is attained but with a curved (cellular) flame front. The critical range for the non-steady propagation is also found to be a function of the Lewis number of the deficient reactant.
\end{abstract}

Key words: spray flame, oscillatory combustion

AMS subject classification: 80A25, 80A32

\section{Introduction}

It is remarkable that, despite the widespread utilization of liquid fuels supplied as a spray of droplets in many practical combustion applications, there are certain basic physical phenomena associated with such two-phase liquid spray-gas systems that have not been thoroughly investigated. Thus, there is experimental evidence [1-7] that the burning velocity of a flame propagating through a mixture of air and mono-sized fuel droplets can exceed that of a flame propagating through an

*Corresponding author. E-mail: grishas@post.tau.ac.il 
equivalent fuel vapor/air mixture. Furthermore, it is possible to identify an optimum droplet size at which the flame speed achieves a maximum value. For off-stoichiometric fuel lean mixtures Atzler [8] observed that premixed laminar spray flames propagate in a pulsating manner alternating through periods of flame front acceleration and deceleration. These pulsations are accompanied by synchronous flame front cellularization and smoothing, respectively. For off-stoichiometric fuel rich mixtures laminar spray flames were found to exhibit cellular structures.

In the introduction to a previous study [9] a full, chronologically arranged, account of experimental and theoretical studies on laminar premixed spray flames was given and the interested reader is referred to this reference and the references therein. That study concentrated on fuel rich laminar premixed spray flames and its purpose was to attempt to replicate some of their aforementioned peculiar phenomena by numerical means. Whereas previous stability analysis [10-13], aimed at predicting mathematically the aforedescribed cellularization and pulsating behavior, were predicated on linearization, the numerical study of [9] dispensed with this restriction by retaining the important non-linear terms and was indeed able, in a limited qualitative way, to predict what had been previously observed independently in the laboratory.

The current work is a continuation of [9] in which it was found that the rich premixed laminar spray flame propagates in a steady manner and, depending on the operating conditions, the reaction fronts are eventually either plane or curved. Before a flame reaches its final steady state its velocity was found to oscillate around a certain positive mean value. An important spray-related parameter is the evaporation Damköhler number, being the ratio of a characteristic flow time to a characteristic evaporation time. In [9] values of the evaporation Damköhler number, $\Delta$, examined were restricted to the range $2 \leq \Delta \leq 50$. For this range there is little direct interaction between the evaporation and the reaction front (see the bottom right hand figure in Fig. 1 in which the concentration of fuel vapor and the reaction rate are plotted for one-dimensional premixed spray flames as functions of distance). It is clear that for $\Delta \geq 2$ the fuel completely evaporates ahead of the reaction zone. In this situation, it is natural that the width of the evaporation zone does not impact significantly on the combustion. In fact, laser Schlieren images in Atzler's experimental work [8] seem to indicate that this is precisely the situation that he examined.

In contrast, in the present research we extend our previous study and focus on fuel rich spray pre-mixtures with a vaporization Damköhler number significantly smaller than 2 . This range of values corresponds to liquid fuels of low volatility for which droplets are actually traversed by the reaction zone and continue to evaporate in the oxygen-free region behind it (see Fig. 1).

\section{Mathematical Model}

A conventional constant density reaction-diffusion model is adopted. The chemical kinetics is described using a single-step irreversible exothermic reaction. For the off-stoichiometric fuel-rich case this implies that a conservation equation need only be solved for the deficient reactant, the oxidant. The spray of evaporating droplets is modeled using the sectional approach [14] and under the assumption that the liquid fuel mass fraction is small (typically of the order of $10^{-2}$, at most). 

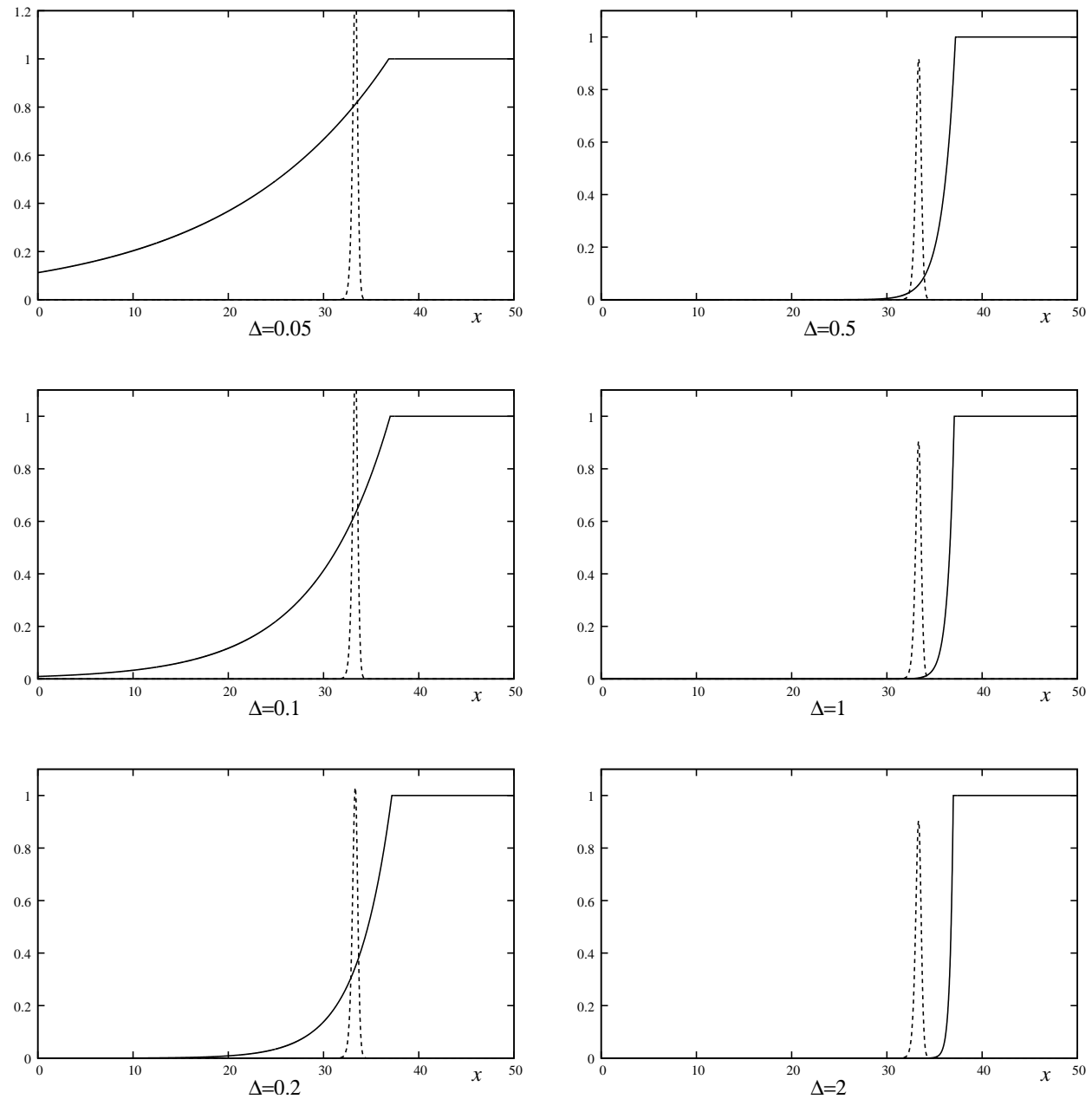

Figure 1: Liquid fuel mass fraction (solid line) and reaction rate (broken line) in rich laminar premixed spray flames for different evaporation Damköhler numbers $(L e=0.85)$ 
For the sake of simplicity the mono-sectional case is considered here.

In appropriately chosen units the corresponding set of equations for the temperature, oxidant mass fraction and fuel liquid mass fraction reads

$$
\begin{gathered}
T_{t}=\nabla^{2} T+(1-\sigma) \Omega-\beta S_{v} H\left(T-T_{v}\right) \\
C_{t}=L e_{F}^{-1} \nabla^{2} C-\Omega \\
\left(C_{d}\right)_{t}=-S_{v} H\left(T-T_{v}\right)=-\Delta C_{d} H\left(T-T_{v}\right) \\
\Omega=\frac{1}{2}(1-\sigma)^{2} N^{2} L e^{-1} C \exp \left(N\left(1-\frac{1}{T}\right)\right)
\end{gathered}
$$

In these equations $T$ is the non-dimensional temperature in units of $T_{b}=T_{0}+Q C_{0} / c_{p}$, the adiabatic temperature of combustion products in the pure gaseous case, $C$ and $C_{d}$ are the scaled concentrations of the oxidant mass fraction and fuel liquid mass fraction in units of $C_{0}$ which is the oxidizer concentration in the fresh mixture. In the fresh premixture: $C=C_{0}, C_{d}=C_{d 0} ; x$, $y$ are the non-dimensional spatial coordinates in units of $l_{t h}=D_{t h} / U_{b}$, the thermal width of the flame, $D_{t h}$ is the thermal diffusivity of the mixture, $U_{b}$ is the burning velocity of a planar adiabatic flame in the pure gaseous case, $t$ is the non-dimensional time in units of $l_{t h} / U_{b} ; \sigma=T_{0} / T_{b}$, with $T_{0}$ the fresh mixture temperature, $N=T_{a} / T_{b}$ is the non-dimensional activation energy and $T_{a}$ is the activation temperature, $T_{v}=T_{V} / T_{b}$ where $T_{V}$ is the boiling temperature of the fuel, $L e=D_{t h} / D$ is the Lewis number, $D$ is the molecular diffusivity of the oxygen, $c_{p}$ is the specific heat, $Q$ is the heat of reaction, $\Omega(C, T)$ is the appropriately normalized reaction rate to ensure that at large $N$ the non-dimensional speed of a steadily propagating planar adiabatic flame is close to unity in the pure gaseous mixture, $\Delta=\left(\frac{D_{t h}}{U_{b}^{2}}\right) \hat{C}$ is the vaporization Damköhler number, $\hat{C}$ is the sectional vaporization coefficient and, finally, $\beta$ is the nondimensional latent heat of vaporization of the droplets in the spray. All parameters employed are assumed to be temperature independent. In addition, use is made of the Heaviside function,

$$
H(t)=\left\{\begin{array}{ll}
0 & t<0 \\
1 & t \geq 0
\end{array}\right. \text { Heaviside function }
$$

to denote the onset of appreciable evaporation when the spray's droplets attain the boiling point of the liquid fuel.

Equations (2.1)-(2.3) are to be solved in the two-dimensional region, $0<x<L$ and $-\infty<$ $y<\infty$ subject to the boundary

$$
T_{x}=C_{x}=0 \quad \text { at } x=0, L
$$

The flame is assumed to propagate upward. Hence the boundary conditions at $y= \pm \infty$,

$$
\begin{array}{crl}
T=\sigma, C=C_{0}, \quad C_{d}=C_{d 0} & \text { at } y \rightarrow+\infty \\
T_{y}=C_{y}=0 & \text { at } y \rightarrow-\infty
\end{array}
$$


Initial conditions contain a small temperature perturbation,

$$
\begin{array}{cr}
T=\sigma, C=C_{0}, \quad C_{d}=C_{d 0} & \text { at } y>0 \\
T=1+0.01(x-L / 2), C=0, \quad C_{d}=0 & \text { at } y<0
\end{array}
$$

The problem (2.1)-(2.7) is solved for $N=10, L e=0.8,0.85,0.9, \sigma=0.11-0.2, L=100$, $\Delta=10, T_{v}=0.169, \beta=0.041$. The computational strategy involves using finite differences and the solution of the resulting difference equations via the alternating direction implicit method (see [15]).

\section{Results}

In [9] the sensitivity of premixed spray flames to the Lewis number was demonstrated and compared to that of purely gaseous flames. Some results are presented in Table 1. For $L e=0.85$ it was shown that the gas flame is stable whereas the equivalent spray flame is unstable. For this reason the value of the Lewis number was first taken to be 0.85 .

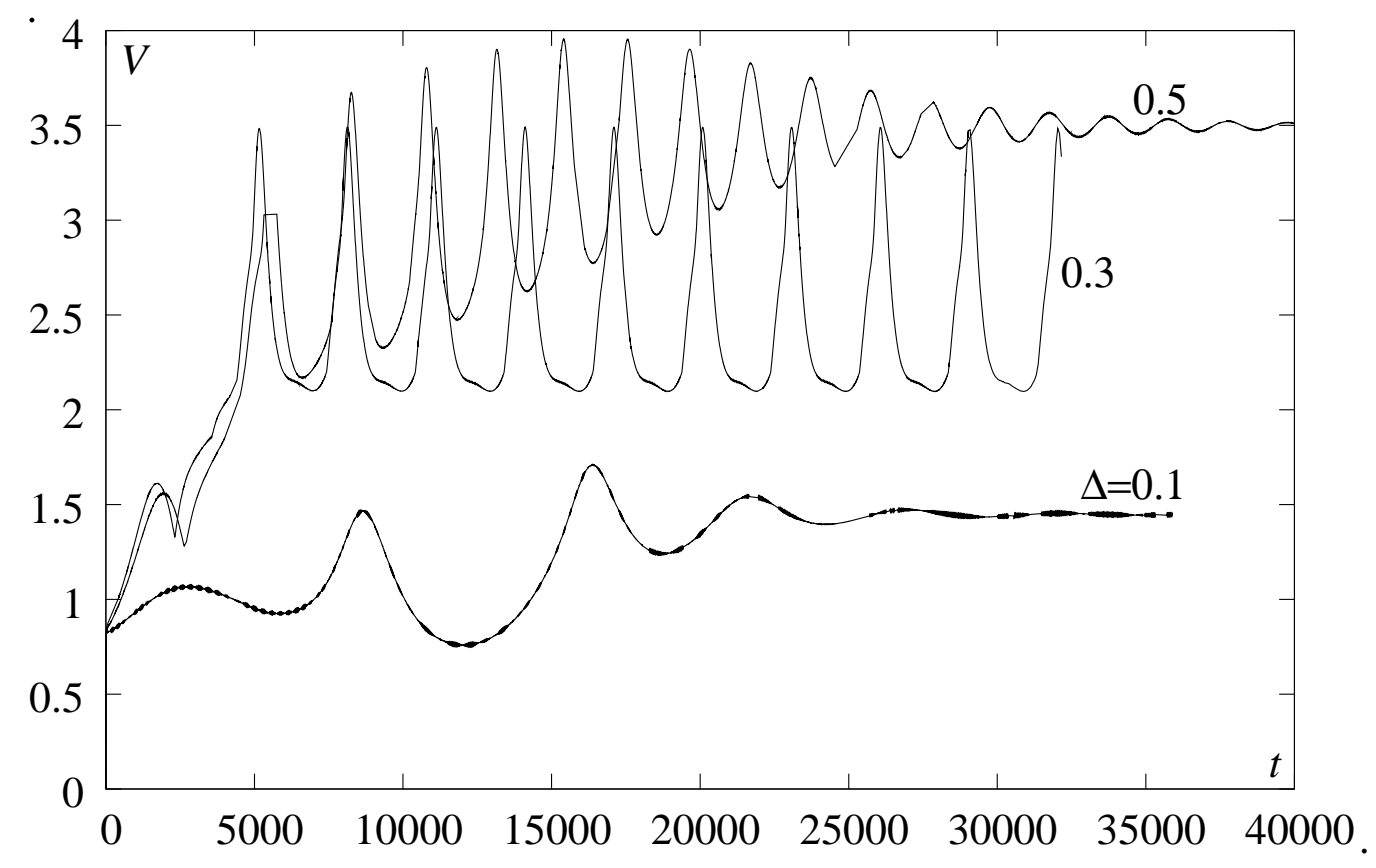

Figure 2: Flame front amplitude $\delta$ as a function of time $t$ for several values of the vaporization Damköhler number $\Delta=0.1,0.3,0.5$ with $L e=0.85$

The main results obtained from the current numerical simulations are shown on Fig. 2 where the flame front amplitude, $\delta(t)=\max _{0 \leq x \leq L}(y(x, t))-\min _{0 \leq x \leq L}(y(x, t))$ where $y(x)$ is the flame front, is 
plotted as a function of time. One can see that there is a range of values of $\Delta$ for which the spray flame propagates in an oscillatory manner.

\begin{tabular}{|c|c|c|}
\hline & $C_{d 0}=0$ & $C_{d 0}=1$ \\
\hline$L e=0.8$ & unstable & unstable \\
\hline$L e=0.85$ & stable & unstable \\
\hline$L e=0.9$ & stable & stable \\
\hline
\end{tabular}

Table 1: Sensitivity of Gas Flame and Spray Flame to Lewis number (for vaporization Damköhler number=10).

For $\Delta=0.2$ (not shown here) and 0.3 oscillations occur. The simulations with vaporization Damköhler numbers outside the interval, namely $\Delta=0.1,0.4$ and 0.5 , lead to stable propagation of a curved reaction front with results similar to those described in [9].

The impact of spray evaporation on rich flame propagation is via the heat consumed by the liquid drops for evaporation. It is of utmost importance where this phenomenon takes place. For a high enough Damköhler number $\Delta=0.5$ evaporation and heat losses occur in front of the reaction zone (see Fig. 1), whereas for small $\Delta=0.1$ the evaporation takes place mainly after the flame front (Fig. 1). Only for the narrow interval $0.2 \leq \Delta \leq 0.3$ the heat losses compete and interact directly with the exothermic chemical reaction heat production and the interplay produces oscillations.

Figure 3 shows the flame front propagation during one period of oscillation. Here, for clarity of presentation, we define the flame front position as an isoline of deficient reactant (oxygen concentration) equal to 0.1 and, in addition, stretch the flame front with the help of the following transformation

$$
y^{\prime}(x)=\bar{y}+100(y(x)-\bar{y}) \text { where } \bar{y}=\frac{1}{L} \int_{0}^{L} y(x, t) d x .
$$

It is clearly seen that there is galloping propagation even for this rich spray flame, as was found experimentally for lean spray flames. Moreover the unsteady propagation arises only in twodimensional modeling. The corresponding one-dimensional simulation ends up with steady propagation of the reaction wave for all studied vaporization Damköhler numbers.

During the period of oscillation, (Fig. 3) the number of cells comprising the flame front changes. At the beginning and end of the single cycle there are two cells, whereas in the middle there is a single cell. This periodic change of the number of the cells results in the continuing oscillatory propagation (see Fig. 2).

Figure 4 shows a graph of the average velocity of the flame front for different values of the evaporation Damköhler number. This coincides with the results of a one-dimensional simulation, despite the absence of flame curvature and unstable propagation. An explanation of this phenomenon is proffered in [9]. Fig. 4 clearly demonstrates that for small $\Delta$ the flame velocity is relatively large. In this case evaporation occurs far after the reaction zone, and the spray-induced 


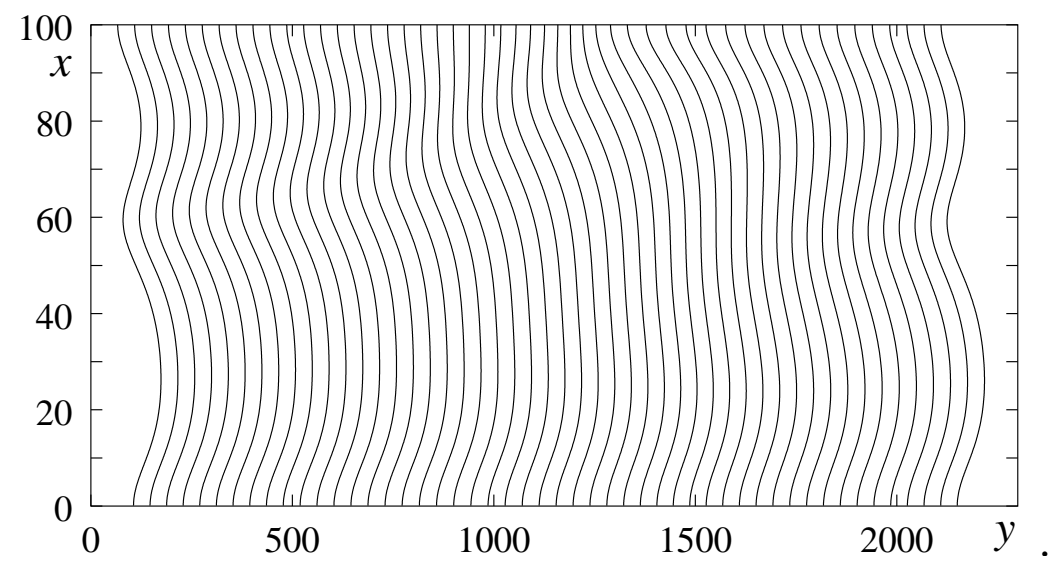

Figure 3: Positions of the flame front (propagating from left to right) at several equidistant moments, for a single period of oscillations $(\Delta=0.3, L e=0.85)$

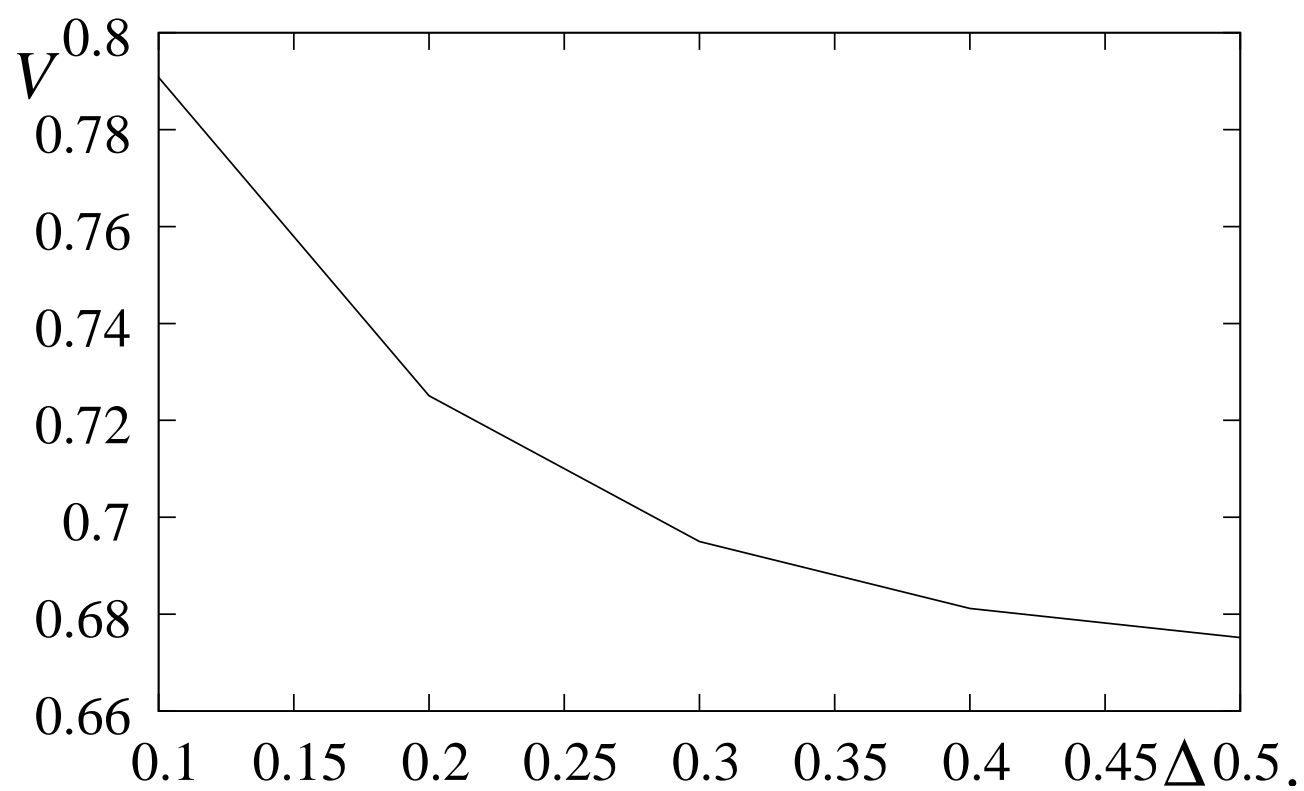

Figure 4: Average velocity of rich spray flame propagation as a function of the vaporization Damköhler number with $L e=0.85$ 
heat losses do not strongly challenge the exothermic reaction rate. However, if the evaporation Damköhler number is high enough then the evaporation consumes heat ahead of the chemical reaction. This results in a drastic reduction of the average flame velocity which becomes almost independent of $\Delta$.

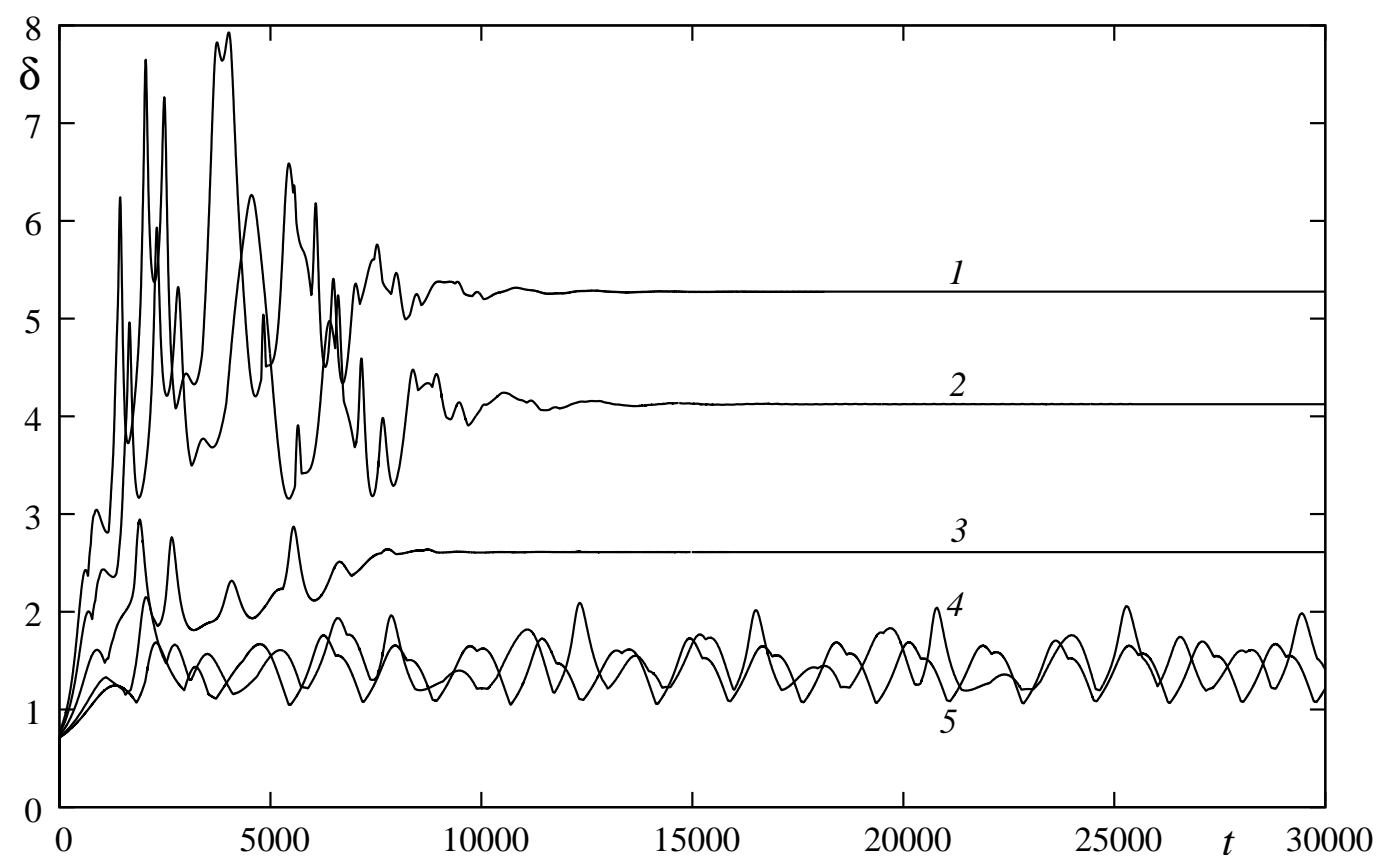

Figure 5: Flame front amplitude $\delta$ as a function of time $t$ for several values of Damköhler number $\Delta=0.2(1), 0.1(2), 0.05(3), 0.02(4), 0.01(5)$ with $L e=0.8$

Now suppose the Lewis number is 0.8 . Referring to Table 1 it is seen that, for a vaporization Damköhler number of 10 both the spray flame and its gaseous equivalent are unstable. In the current study the results obtained are shown in Fig.5. In this instance, it is seen that oscillatory flame propagation occurs for smaller values of $\Delta$. Also, the unstable propagation is rather chaotic, not regular. This result is in line with a previous study of cellular pure gaseous flames [16], which may be considered as the limiting case of $\Delta=0$ (see Eq.(2.3)).

\section{Conclusions}

In a new numerical study of laminar rich premixed spray flames it was shown that:

1. There is a narrow band of evaporation Damköhler numbers for which the endothermic heat loss resulting from fuel spray droplets evaporation competes with the exothermic chemical reaction in the gas phase resulting in oscillatory propagation of the spray flame, for $L e=0.85$. For values 
outside this band the flame front admits a cellular structure and attains a constant propagation speed.

2. For $L e=0.8$ there is also a narrow range of (smaller) evaporation Damköhler numbers for which unsteady propagation occurs. In this instance too if the evaporation Damköhler number is sufficiently high the flame admits a curved shape but propagates steadily.

Finally, it is noted that the pulsating propagation of laminar premixed spray flames was so far only observed experimentally for off-stoichiometric lean spray flames. The current work indicates that the phenomenon is not restricted to such pre-mixtures, although it may be surmised that the mechanism responsible for the oscillatory behavior may differ. An investigation of the propagation of lean spray flames is currently under investigation and some preliminary results were recently presented [17].

\section{Acknowledgements}

LSK and GIS gratefully acknowledge the support of the US - Israel Binational Science Foundation (Grant 2006-151), and the Israel Science Foundation (Grant 32/09). JBG thanks the Technion Fund for the Promotion of Research, the Lady Davis Chair in Aerospace Engineering and the US - Israel Binational Science Foundation (Grant 2004-069) for supporting this research.

\section{References}

[1] C.E. Polymeropoulos. Flame propagation in a one-dimensional liquid fuel spray. Combust. Sci. Technol., 9 (1974), 197-207.

[2] C.E. Polymeropoulos, S. Das. The effect of droplet size on the burning velocity of a keroseneair spray. Combust. Flame, 25 (1975), 247-257.

[3] Y. Mizutani, A. Nakajima. Combustion of fuel vapor-drop-air systems: Part I - open burner flames. Combust. Flame, 20 (1973), 343-350.

[4] Y. Mizutani, A. Nakajima. Combustion of fuel vapor-drop-air systems: Part II - spherical flames in a vessel. Combust. Flame, 20 (1973),351-357.

[5] S. Hayashi, S. Kumagai. Flame propagation in droplet-vapor-air mixtures. Fifteenth Symposium (International) on Combustion, The Combustion Institute, Pittsburgh, Penn., (1974), $445-452$.

[6] S. Hayashi, S. Kumagai, T. Sakai. Propagation velocity and structure of flames in dropletvapor-air mixtures. Combust. Sci. Technol., 15 (1976), 169-177. 
[7] D.R. Ballal, A.H. Lefebvre. Flame propagation in heterogeneous mixtures of fuel droplets, fuel vapour and air. Eighteenth Symposium (International) on Combustion, The Combustion Institute, Pittsburgh, Penn., (1981), 321-328.

[8] F. Atzler. Fundamental studies of aerosol combustion. Ph.D. Thesis, School of Mechanical Engineering, University of Leeds, Leeds, U.K., 1999.

[9] J.B Greenberg J.B., L.S. Kagan, G.I. Sivashinsky. Stability of rich premixed spray flames. Atomization and Sprays, 19 (2009), No. 9, 863-872.

[10] J.B. Greenberg, A.C. McIntosh. J. Brindley. Linear stability analysis of laminar premixed spray flames. Proc. R. Soc. Lond. A, 457 (2001), 1-31.

[11] J.B. Greenberg. Stability boundaries of laminar premixed polydisperse spray flames. Atomization and Sprays, 12 (2002), 123-143.

[12] C. Nicoli, P. Haldenwang, S. Suard. Analysis of pulsating spray flames propagating in lean two-phase mixtures with unity Lewis number. Combust. Flame, 143 (2005), 299-312.

[13] C. Nicoli, P. Haldenwang, S. Suard. Effects of substituting fuel spray for fuel gas on flame stability in lean premixtures. Combust. Flame, 149 (2007), 295-313.

[14] J.B. Greenberg, I. Silverman, Y. Tambour. On the origins of spray sectional conservation equations. Combust. Flame, 93 (1993), 90-96.

[15] L. Kagan, G. Sivashinsky. Flame propagation and extinction in large-scale vertical flows. Combust. Flame, 120 (2000), 222-232.

[16] L. Kagan, G. Sivashinsky. Self-fragmentation of nonadiabatic cellular flames. Combust. Flame 108 (1997), 220-226.

[17] L.S. Kagan, J.B. Greenberg, G.I. Sivashinsky, Propagation of lean premixed spray flames. Presented at the $4^{\text {th }}$ European Combustion Meeting, Vienna, Austria, April, 2009. 